\title{
Sistema multipotenciostato basado en instrumentación virtual
}

\author{
Multipotenciostat System Based on Virtual Instrumentation
}

\author{
Arrieta-Almario Álvaro Angel \\ Escuela de Ingeniería y Arquitectura \\ Universidad Pontificia Bolivariana Montería, Colombia \\ Correo: alvaro.arrieta@upb.edu.co
}

\author{
Tarazona-Cáceres Rosa Liliana \\ Escuela de Ingeniería y Arquitectura \\ Universidad Pontificia Bolivariana Montería, Colombia \\ Correo: rosa.tarazona@upbmonteria.edu.co
}

Información del artículo: recibido: marzo de 2012, reevaluado: abril de 2012 y febrero de 2013, aceptado: mayo de 2013

\section{Resumen}

En este trabajo se desarrolló un sistema electrónico multicanal de medidas electroquímicas o multipotenciostato, basado en la técnica de medición de voltametría cíclica, controlado desde una computadora que monitorea, a través de un circuito electrónico, tanto el voltaje generado desde la PC y suministrado a una celda electrolítica, como la corriente que circula a través de los electrodos de trabajo de dicha celda. Se utilizó la instrumentación virtual para diseñar el software de aplicación y la interfaz de usuario, así como los módulos de adquisición de datos NI 9263 y NI 9203 de National Instruments @ para la comunicación entre el circuito multipotenciostato y el software diseñado. El sistema se probó para una sustancia con una propiedad redox conocida y también se utilizó para la discriminación y clasificación de muestras de café.

\footnotetext{
Abstract

To carry out this project an electronic multichannel system of electrochemical measurement or multipotenciostat was developed. It is based on the cyclic voltammetry measurement technique, controlled by a computer that monitors, by means of an electronic circuit, both the voltage generated from the Pc and supplied to an electrolytic cell, and the current that flows through the electrodes of it. To design the application software and the user interface, Virtual Instrumentation was used. On the other hand, to perform the communication between the multipotenciostat circuit and the designed software, the National Instruments NI9263 and NI9203 acquisition modules were used. The system was tested on a substance with a known REDOX property, as well as to discriminate and classify some samples of coffee.
}

\section{Descriptores:}

- instrumentación virtual

- adquisición de datos

- sistema multicanal

- voltamograma

- multipotenciostato básico
Keywords:

- virtual instrumentation

- data acquisition

- multichannel system

- voltammogram

- basic multipotenciostat 


\section{Introducción}

La combinación de los conocimientos desarrollados sobre áreas del saber específico tales como la química, la electrónica y el desarrollo de la tecnología informática han contribuido al avance científico y tecnológico con la aparición de nuevos sensores, materiales e instrumentos de medición, que utilizan interesantes técnicas de control y monitoreo, como son la nariz electrónica (Persaud y Dodd, 1982; Gardner et al., 2000; Natale et al., 2001) y la lengua electrónica (Lvova et al., 2002; Winquist et al., 1997; 1999). El interés por este último sistema ha crecido notablemente, no solo por su potencial, variedad en los usos, y por que los resultados no son esencialmente aplicables a la sensación del gusto, sino también pueden estar relacionados con una propiedad de calidad de la muestra.

Las lenguas electrónicas se están convirtiendo en dispositivos de análisis cualitativo y cuantitativo para soluciones complejas, ya que pueden llegar a catalogar diferentes tipos de muestras, detectar sustancias que alteren la calidad de determinados alimentos, como contaminantes, impurezas y sustancias tóxicas (Vlasov et al., 2002; Parra et al., 2006a).

Las lenguas electrónicas están formadas por un sistema automático de control, una matriz de sensores químicos, un sistema de adquisición de datos y un software con el algoritmo apropiado para el procesamiento de la señal obtenida y, según se requiera, un análisis cualitativo o cuantitativo de la muestra analizada (Jiménez et al., 2002). El principal criterio de aplicación y desarrollo del conjunto de sensores para una lengua electrónica es su alta sensibilidad y selectividad cruza$\mathrm{da}$, esta última se puede entender como la capacidad que tiene un sensor de responder de manera reproducible a un número de compuestos diferentes de una solución (Jiménez et al., 2002; Vlasov, 1988).

Para mejorar las aplicaciones analíticas de los sensores electroquímicos, investigadores a nivel internacional han desarrollado matrices de sensores con sofisticadas técnicas de procesamiento para interpretar los resultados. Parte de estos estudios se han realizado en los polímeros conductores como el polipirrol, gracias a su buena conductividad eléctrica, estabilidad del medio ambiente, buena solubilidad, propiedades redox y la relativa facilidad de oxidación del monómero (Arrieta y Tarazona, 2008). Ejemplo de ello son los trabajos realizados por $\mathrm{Pa}$ rra, Arrieta, Fernández, García, Apetrei, Rodríguez y De Saja. Además del interés científico, los polímeros conductores son especialmente estudiados por la variedad de posibles aplicaciones, como la discriminación y clasificación de muestras de café (Arrieta y Osorio, 2010), vino (Parra et al., 2006b), cerveza (Rudnitskaya et al., 2009), entre otros.

Otra característica importante de los sistemas de lenguas electrónicas es que se basan en diferentes técnicas de mediciones electroquímicas, tales como la potenciometría (Lvova et al., 2002; Toko, 1998; Verrelli et al., 2007), la amperometría (Verrelli et al., 2007; Pividori, 2002) o la voltametría (Winquist et al., 1997; Ivarsson et al., 2001; Parra et al., 2005). En la técnica voltamétrica se emplea un sistema de tres electrodos, uno de trabajo, uno de referencia y uno auxiliar. El electrodo auxiliar únicamente permite la circulación de la corriente hacia el electrodo de trabajo. El electrodo de referencia debe conectarse a una entrada de alta impedancia del sistema de medición de forma que no se produzca circulación de corriente por el mismo. El electrodo de trabajo es el electrodo sobre el que se quieren realizar las medidas. El objetivo es poder aplicarle un potencial controlado (V) en función del tiempo (t) y medir la corriente (I) que circula por él. La técnica se puede describir como una función de $\mathrm{V}$, I y t, observándose en un gráfico resultante corriente-potencial llamado voltamograma (Kounave, 1997).

Para la utilización de las diferentes técnicas electroquímicas, es indispensable contar con un sistema electrónico capaz de generar las señales de voltaje, aplicar estas señales a la celda electroquímica, recoger los datos de corriente que se generan y almacenarlos para su posterior tratamiento. Básicamente, se emplean instrumentos comerciales, denominados potenciostatos, modificados o desarrollos realizados en laboratorios que siguen los modelos comerciales, como se reporta en los trabajos realizados por Parra, Arrieta, Fernández, García, Apetrei, Rodríguez y De Saja, donde las mediciones electroquímicas se llevaron a cabo con el potenciostato galvanostato comercial (Parra et al., 2006). Una de las principales virtudes de desarrollar el sistema de medida es que permite tener más control sobre las prestaciones del instrumento y manejar mejor el acoplamiento con la red de sensores y el software de control y tratamiento de datos, llegando a ser más flexible, portátil, de fácil manejo para el usuario y económico, además de que posea la precisión y exactitud de los equipos comerciales, de tal manera que la medida que se obtenga posea la misma calidad y confianza.

La incursión de nuevas tecnologías como la instrumentación virtual y las tarjetas de adquisición de datos (DAQ, Data Acquisition Systems) llevan a cabo esta tarea de una forma más sencilla gracias a sus características y prestaciones. Entre las que se encuentra la facilidad de crear una interfaz hombre máquina "HMI" (Human Machine Interface), manejo de funciones de análisis y 
procesamiento de señales, códigos eficientes para el almacenamiento de datos, la comunicación con otros equipos y la adquisición de señales. Lo que posibilita diseñar un dispositivo versátil, como lo reporta Lopes C. Juliana (Lara y Pelegri, 2007; Calobozo, 2004).

En este trabajo se presentan los resultados de diseño e implementación de un sistema de medición electrónico multicanal o multipotenciostato, manipulado desde una computadora, que utiliza la combinación de un hardware y software para realizar las medidas tanto de voltaje suministrado a una celda electroquímica, como la corriente que circula a través de los electrodos de trabajo de dicha celda, a través DAQ. Para el desarrollo del software se escogió la programación en Labview 2009 ( , que utiliza aplicaciones conocidas como Instrumentos Virtuales VI (Virtual Instruments) (Lajara y Pelegrí, 2007), para manipular las variables de voltaje, corriente y registrar los voltamogramas requeridos. La técnica electroquímica utilizada fue la voltametría cíclica, en un rango de barrido entre $-2 \mathrm{~V} \mathrm{y}+2 \mathrm{~V}$.

\section{Materiales y métodos}

Para el diseño del sistema multicanal se integran tres elementos: la implementación del multipotenciostato básico, la adquisición de datos y la instrumentación virtual.

\section{Multipotenciostato y etapa de adquisición de datos}

El potenciostato es un instrumento de monitoreo electroquímico que controla la diferencia de potencial entre un electrodo de trabajo y un electrodo de referencia ubicados dentro de una celda electroquímica. Básicamente, está constituido por un bloque de control de voltaje y uno de medida de corriente. El primer bloque debe garantizar que no circulará ninguna corriente por el electrodo de referencia, para ello se utiliza un amplificador de alta impedancia en configuración seguidor de voltaje, conectado al electrodo de referencia. Además este bloque es el encargado de aplicar, entre el electrodo de referencia y trabajo, el voltaje establecido por la técnica electroquímica a utilizar. El segundo bloque debe generar un voltaje que sea proporcional a la corriente que circula por el electrodo de trabajo y debe garantizar que no haya circulación de corriente por el electrodo de referencia (Kounave, 1997). El multipotenciostato tiene el mismo principio, pero cuenta con varios electrodos de trabajo y por ello presenta mayor eficiencia en las medidas tomadas.

El funcionamiento de la aplicación realizada depende de la técnica seleccionada, en este caso la voltametría cíclica. El usuario debe establecer en la HMI, diseñada para la aplicación, parámetros como la velocidad de barrido y los valores inicial, máximo y mínimo de la señal, junto con el resto de datos necesarios para configurar cada ensayo que se quiera hacer. Los valores de voltaje se transmiten por el puerto USB al equipo de medida, este aplica la señal al electrodo de trabajo y muestrea las señales de corriente, las cuales se envían a la computadora donde son representadas gráficamente y se almacenan.

El esquema simplificado del sistema multicanal diseñado se muestra en la figura 1 .

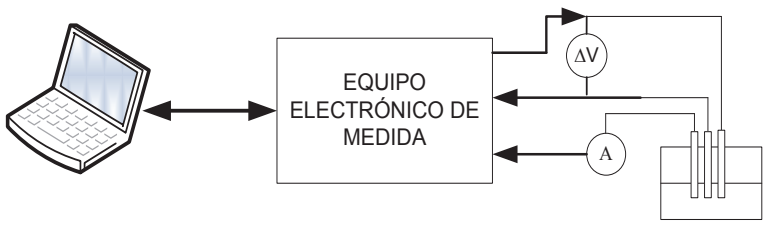

Figura 1. Diagrama esquemático del sistema multicanal

El equipo electrónico de medida está constituido por los módulos de adquisición de datos y un multipotenciostato básico, este último conformado por amplificadores operacionales que realizan el control de voltaje de la técnica electroquímica utilizada. La señal proveniente de la computadora se lleva al equipo de medida a través del módulo de adquisición y este lo entrega al bloque analógico de los amplificadores operacionales, que a su vez, lo lleva a la celda electroquímica, donde se encuentran los electrodos de referencia, auxiliar y electrodos de trabajo. Una vez aplicada la señal de voltaje a la celda, se inicia la reacción química y el equipo, a través del módulo de adquisición empieza a capturar las señales de corrientes generadas de la reacción química, las cuales son proporcionales a la concentración de la sustancia y se entregan a la PC para almacenarlas. Con el voltaje generado de la técnica de voltametría cíclica y la corriente detectada, en el software de aplicación diseñado se generan las respectivas gráficas de los voltamogramas para cada electrodo de trabajo.

Como las señales analógicas de voltaje y corriente se manejan en una computadora, fue necesario elegir y diseñar una circuitería que realizara los ajustes para el acoplamiento de las señales. Se utilizaron los módulos NI CompactDAQ USB de National Instruments, que presentan una circuitería compacta y robusta, con todo lo necesario para el correcto acoplamiento de señales analógicas digitales sin necesidad de circuitería externa. El bloque de los módulos de adquisición de la National Instruments utilizado está conformado por el modulo NI cDAQ-9174, DAQ NI9263 y DAQ NI9203 (figura 2). 


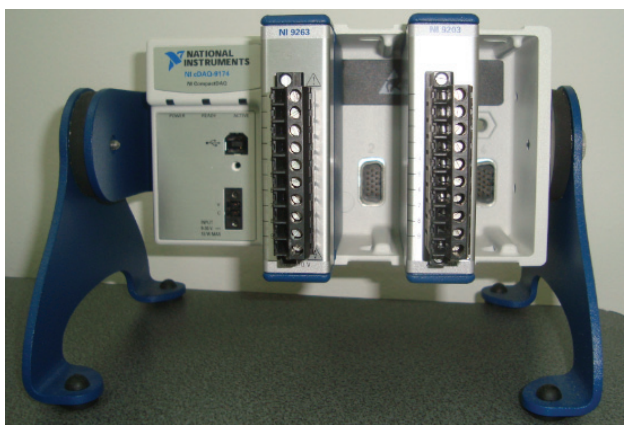

Figura 2. Módulos de adquisición de datos

El NIcDAQ-9174 es un chasis NI CompactDAQ USB 2.0 de alta velocidad, de resolución 32 bits, con 4 ranuras, diseñado para sistemas pequeños y portátiles de pruebas de medidas mixtas. Se puede combinar con hasta cuatro módulos de E/S para un sistema de entrada analógica, salida analógica, E/S digital (National Instruments Datasheet, 2009a). Este módulo recibe la señal de la técnica voltametría cíclica generada de la computadora y lleva la señal de corriente generada en la celda electroquímica a la PC.

El módulo DAQ NI9263 es un módulo de adquisición de datos de salida analógica, de $100 \mathrm{kS} / \mathrm{s}$ (kilo muestras por segundo), con un rango de voltaje de $\pm 10 \mathrm{~V}$, cada canal tiene un convertidor digital analógico (National Instruments Datasheet, 2009b). Se utilizó uno de los cuatro canales para proporcionar la señal de voltaje de la técnica de voltametría cíclica utilizada, a la celda electroquímica. Este módulo lleva la señal de voltaje proveniente de la PC al bloque analógico integrado por amplificadores operacionales. En la figura 3 se muestra el diagrama interno del NI 9263, especificado en el Datasheet "Operating Instructions And Specifications NI 9263".

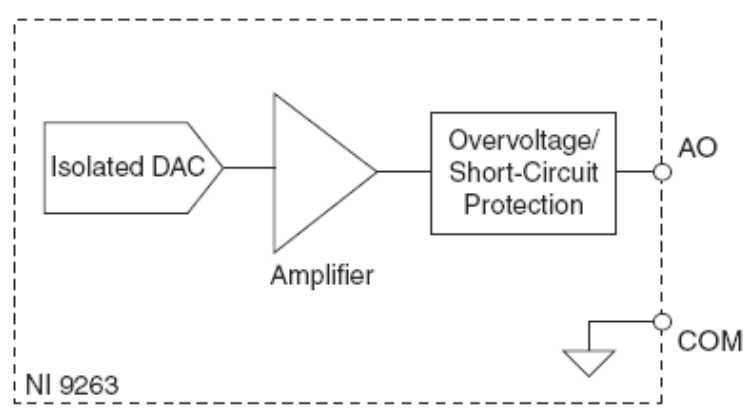

Figura 3. Salida del circuito para un canal de la NI9263 (National Instruments Datasheet, 2009b)
El módulo DAQ NI9203 contiene ocho canales de entrada analógica de corriente con intervalos programables de $\pm 20 \mathrm{~mA}$, las señales son almacenadas, acondicionadas y muestreadas por un convertidor analógico digital (ADC) de 16 bits de resolución y una velocidad de muestreo total de $200 \mathrm{kS} / \mathrm{s}$ (National Instruments Datasheet, 2008). Este módulo se encarga de capturar la corriente generada en la celda electroquímica, y a través del NIcDAQ-9174 los datos son llevados a la PC. En la figura 4 se muestra el diagrama interno del NI 9203, especificado en el Datasheet "Operating Instructions And Specifications NI 9203".

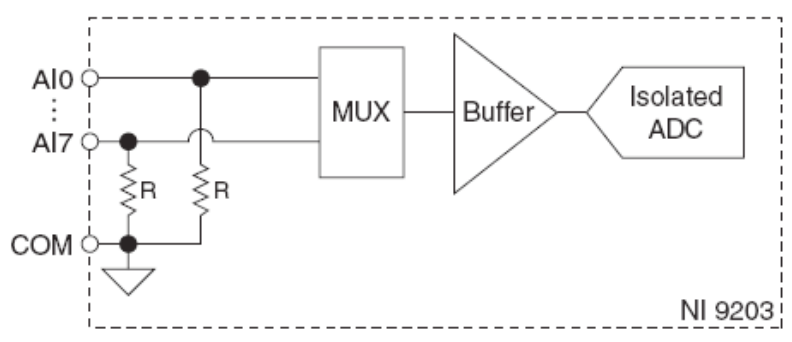

Figura 4. Salida del circuito para un canal de la N19063 (National Instruments Datasheet, 2008)

Las variables tratadas por el sistema multicanal son analógicas por lo que es necesario implementar un circuito utilizando dispositivos apropiados y con características especiales para poder controlar y medir apropiadamente las variables en cuestión. Los amplificadores operacionales (AO), gracias a sus características y prestaciones, son los dispositivos utilizados en esta clase de instrumento. El circuito multipotenciostato básico implementado está conformado por dos $\mathrm{AO}$ de referencia OP77 (Analog devices, 2010) y se muestra en la figura 5.

Dentro del funcionamiento de un potenciostato, se debe garantizar que no habrá circulación de corriente por el electrodo de referencia. Gracias a las características de impedancia de entrada infinita e impedancia de salida casi nula de la configuración de seguidor de voltaje del OP77 (2) (figura 5), se evita que la corriente que empieza a circular en la celda electroquímica lo haga a través del electrodo de referencia y lo pueda polarizar, ocasionando inestabilidad entre el potencial del electrodo de referencia y el electrodo de trabajo.

Además, el potenciostato debe aplicar, entre los electrodos de referencia y trabajo, el voltaje establecido de la voltametría cíclica. En la figura 5 el amplificador OP77 (2), proporciona la diferencia de voltaje entre el electrodo de referencia y el electrodo de trabajo y la lleva al amplificador OP77 (1), el cual toma la información 


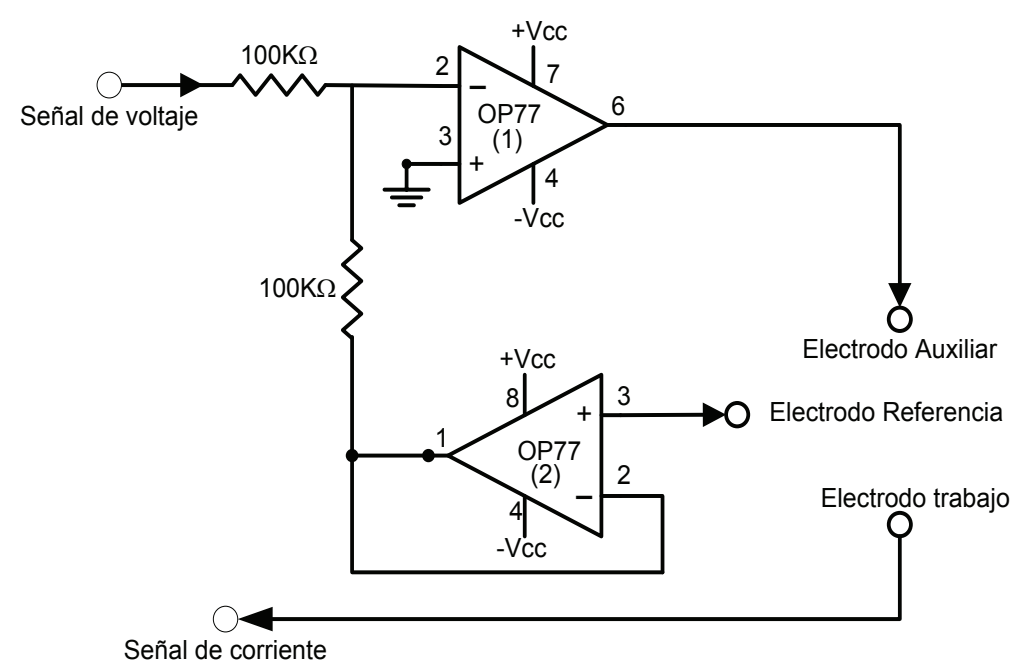

Figura 5. Multipotenciostato básico

y la compara con el voltaje de entrada para ajustar el voltaje del electrodo auxiliar. A su vez, en este último electrodo se genera una corriente que llega al electrodo de trabajo logrando que el potencial entre el electrodo de referencia y el de trabajo se igualen al potencial de entrada.

El OP77 (1) tiene una configuración de amplificador inversor con realimentación negativa, conformada por la impedancia del electrodo de referencia, la impedancia del electrodo auxiliar, la resistencia de $100 \mathrm{k} \Omega$ (2) y el amplificador operacional OP77 (2). Tomando la configuración de realimentación negativa y los AO como ideales, se puede aplicar que para el OP77 (1) en la terminal negativa existe un corto circuito virtual, es decir, un punto de conexión virtual donde el voltaje de entrada al terminal positivo es igual al voltaje de entrada del terminal negativo, en otras palabras a cero voltios, por lo tanto es un punto en el que se suman las señales de salida y entrada, lo cual permite simplificar el análisis de circuitos con $\mathrm{AO}$ y considerar así, desde el punto de vista electrónico, que el electrodo de trabajo también se encuentra a tierra virtual, es decir se encuentra a cero voltios con respecto al potencial del electrodo de referencia. Con esto se puede considerar que al aplicar la señal de voltaje de la técnica electroquímica al electrodo de referencia es como si se aplicara entre el electrodo de referencia y el de trabajo.

Entre los parámetros más importantes a tener en cuenta a la hora de escoger los amplificadores operacionales están: ganancia en lazo abierto alta, una impedancia de entrada alta, una razón de rechazo de modo común alta, una razón de rechazo a la fuente de alimentación alto (es la variación del voltaje de offset respecto a la variación de los voltajes de alimentación, permite rechazar el ruido de una fuente de alimentación), un voltaje de offset bajo y una velocidad de respuesta alta.

Para los amplificadores operacionales OP77 fue necesario diseñar una fuente de polarización de $\pm 5 \mathrm{~V}$ a $400 \mathrm{~mA}$. Se utilizaron capacitores de $4700 \mu \mathrm{F}$ cuya función es eliminar el voltaje de rizado a la salida del puente rectificador y mantener el voltaje constante a la entrada del regulador. También, se utilizaron capacitores de $220 \mu \mathrm{F}$ LowEsr (Equivalent Series Resistance) de baja resistencia, capaces de responder más rápido a picos de corriente y exigencia de los A.O; condensadores cerámicos NP0 (de coeficiente de temperatura negativo, positivo, cero) especiales para eliminar el ruido producido por el regulador de voltaje y evitar cualquier oscilación de alta frecuencia y reguladores de voltaje lineales 7805 y 7905 con salida de voltaje fija +5 $\mathrm{V}$ y $-5 \mathrm{~V}$, respectivamente.

Gracias a las características internas y eléctricas de los módulos NI 9203 y NI 9263 y al chasis CompactDAQ USB 9174 utilizados (tabla 1), se realiza el acoplamiento de las señales entre la PC y la circuitería del sistema multicanal sin necesidad de la implementación de circuitos adicionales.

\begin{tabular}{|c|c|c|c|}
\hline Especificaciones & $\begin{array}{l}\text { NI } 9263 \text { Módulo } \\
\text { de salida }\end{array}$ & $\begin{array}{l}\text { NI } 9203 \text { Módulo } \\
\text { de entrada }\end{array}$ & $\begin{array}{c}\text { Compact } \\
\text { DAQ USB } 9174\end{array}$ \\
\hline Número de canales & 4 & 8 & Chasís de 4 ranuras \\
\hline Tipo de medida & voltaje & corriente & $\begin{array}{l}\text { Entrada / salida } \\
\text { analógica y digital }\end{array}$ \\
\hline $\begin{array}{l}\text { Rango de entrada } \\
\text { programable }\end{array}$ & $\pm 10 \mathrm{~V}$ & $\pm 20 \mathrm{~mA}$ & $x^{2}$ \\
\hline Resolución & 16 Bits & 16 Bits & 32 Bits \\
\hline Velocidad de muestreo & $100 \mathrm{kS} / \mathrm{s}$ & $200 \mathrm{kS} / \mathrm{s}$ & --- \\
\hline
\end{tabular}

Tabla 1. Características internas y eléctricas de los módulos NI 9263, 9203 у 9174 

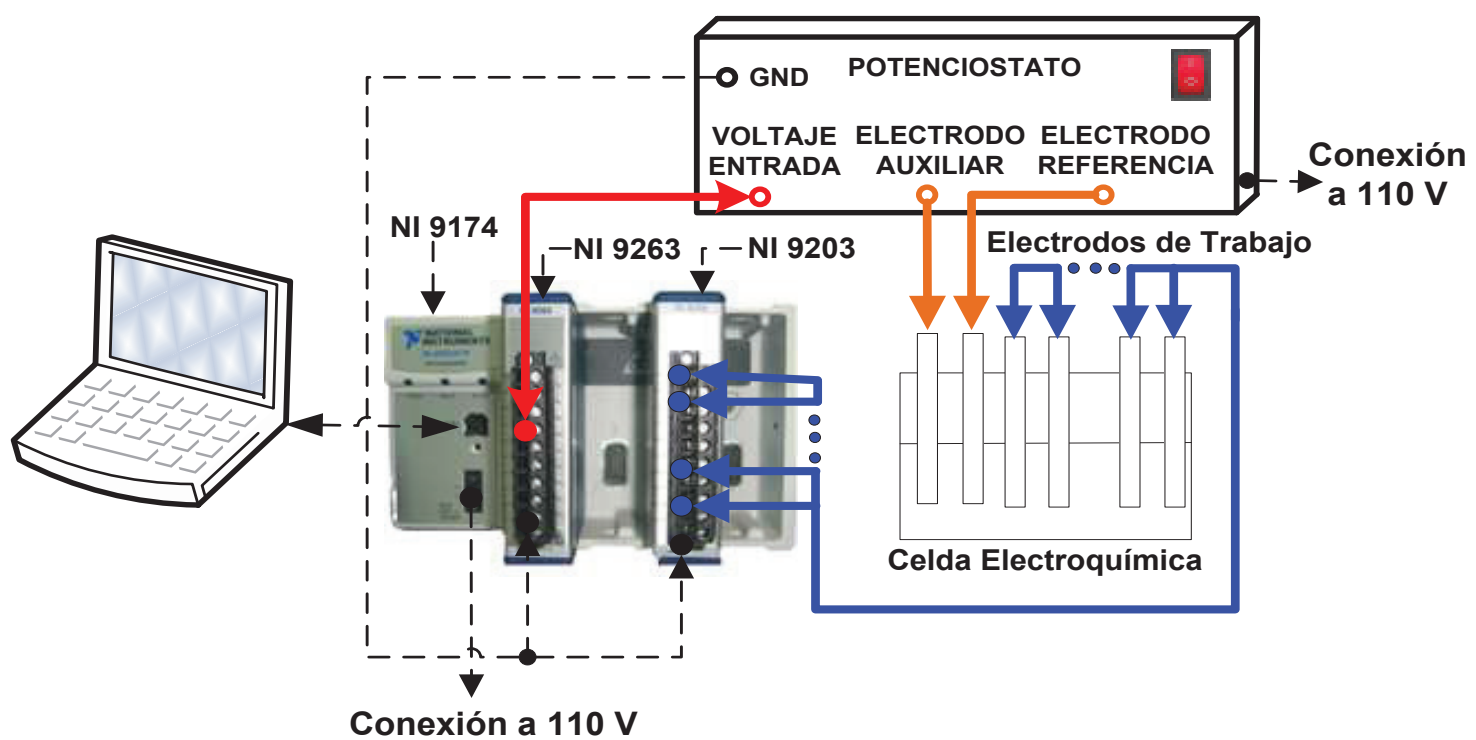

Figura 6a. Conexiones del sistema

En la figura 6a se observan las conexiones de todo el sistema.

\section{Software de aplicación}

En el presente trabajo se desarrolló un sistema multicanal para un prototipo de lengua electrónica, basado en la técnica de voltametría cíclica. Para la generación, almacenamiento, manipulación, procesamiento y presentación de las variables manejadas (voltaje y corriente) se desarrolló un código utilizando el software LabVIEW 2009, se realizó por etapas, las cuales se fueron uniendo a medida que se lograban realizar y simular correctamente. La primera etapa fue el diseño de la señal de la técnica electroquímica, que consistió en generar un voltaje con una forma de onda similar a una escalera típica de la voltametría cíclica. La figura $6 \mathrm{~b}$ muestra la forma de onda generada.

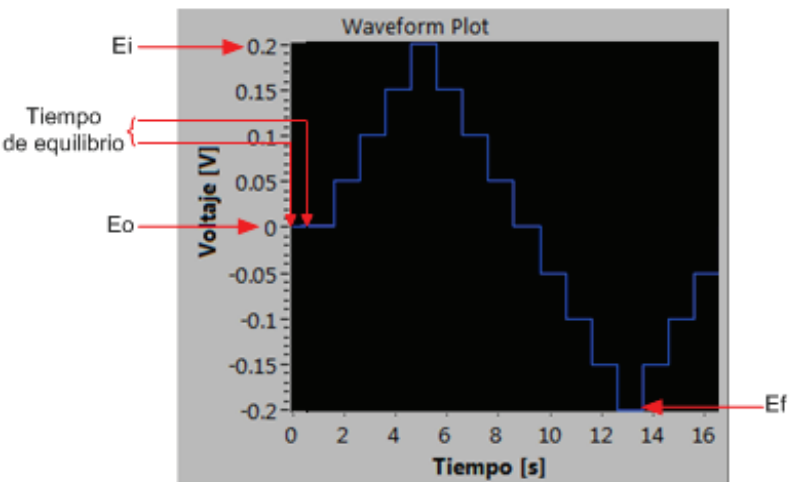

Figura 6b. Onda voltametría cíclica simulada

En la figura 7 se muestra parte del diagrama de flujo utilizado para la onda voltamétrica. 


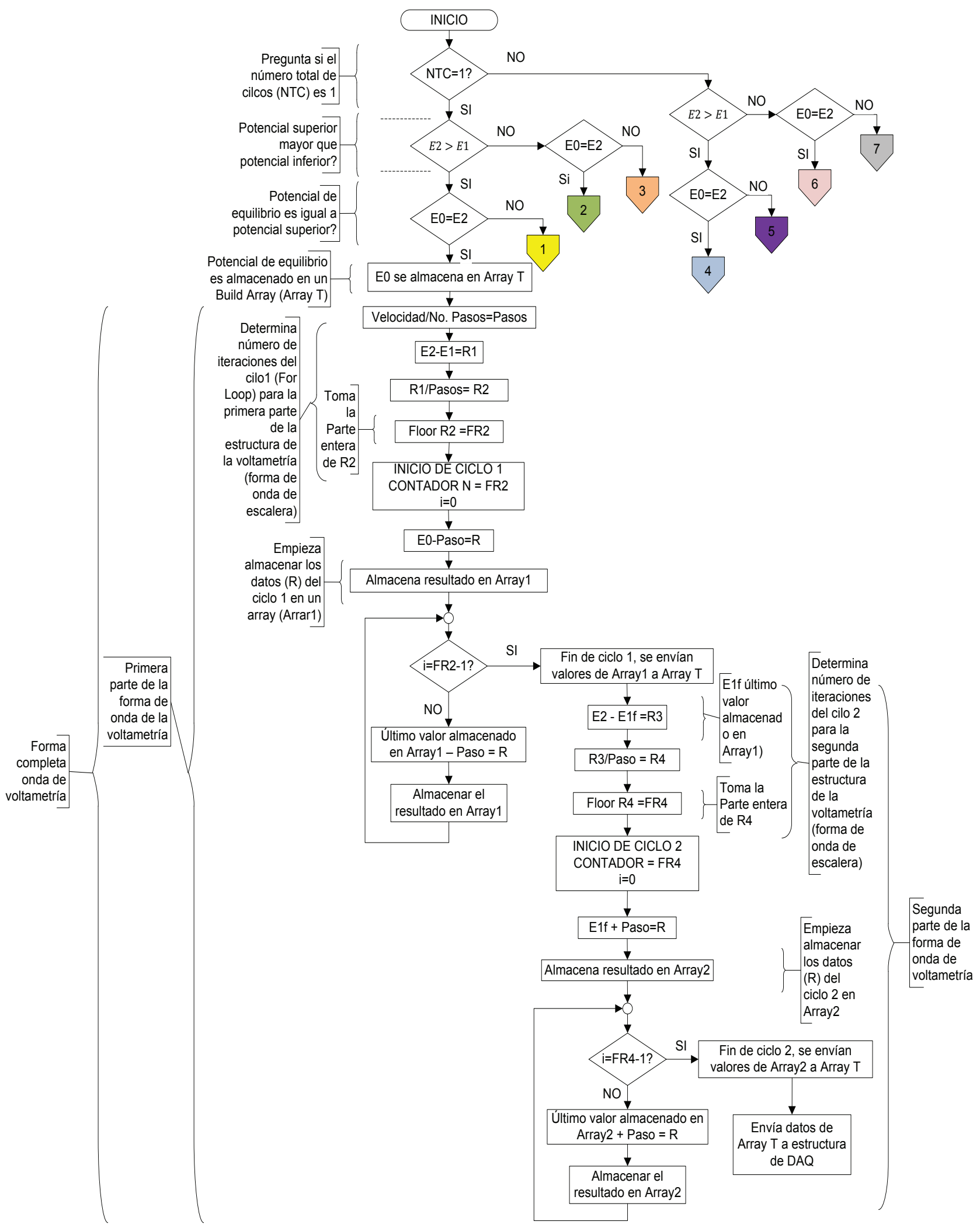

Figura 7. Parte del diagrama de flujo utilizado para la onda voltamétrica 
La segunda etapa fue el diseño de la comunicación entre los módulos de adquisición de datos, DAQ NI9263, DAQ NI9203 y la PC. En las figuras 8 y 9 se observa el código utilizado para cada módulo.

Los datos de corriente adquiridos por el módulo NI9263 se almacenan con los datos de voltaje generados de la técnica voltamétrica. El usuario tiene la posibilidad de guardar un reporte de estos datos en un archivo de hoja de cálculo formato Excel®. En las figuras 10a y $10 \mathrm{~b}$ se muestra la estructura diseñada para la opción de guardar los datos en formato Excel (.xls).

Se creó una HMI para que el usuario configure los parámetros necesarios en el experimento del análisis electroquímico y pueda visualizar los voltamogramas de las señales de interés. En la interfaz se establecen controles, parámetros y registros. Los parámetros son los valores de los potenciales en que se dará el análisis electroquímico, como son: Eo, Ei, Ef, número de ciclos -que se refiere a cuántas veces se repite la forma de onda generada de la voltametría cíclica, tiempo de equilibrio- que es el tiempo de espera para que el potencial Eo se estabilice y en el cual inicializa el experimento. Otros parámetros importantes son la velocidad de barrido o pendiente de la rampa dada en voltios por unidad de tiempo (V/s), el número de pasos o escalones que se dan por voltio, que tiene unidades de pasos por segundo y una base de datos para el electrodo de referencia con su respectivo potencial. En la tabla 2 se muestran los parámetros utilizados con sus respectivos intervalos de uso.

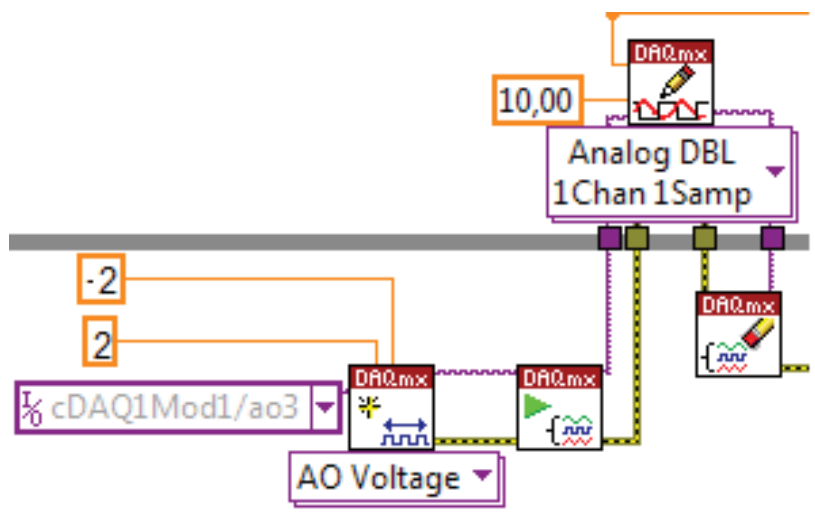

Figura 8. Código de comunicación para el módulo DAQ NI9263

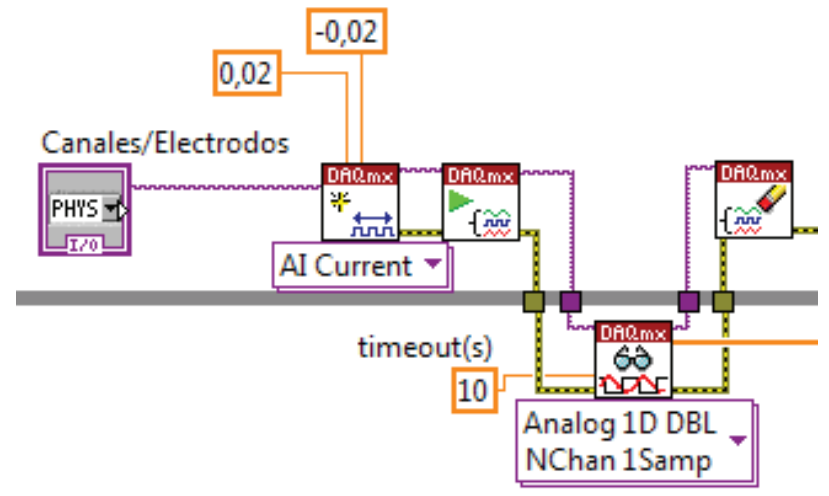

Figura 9. Código de comunicación para el módulo DAQ NI9203

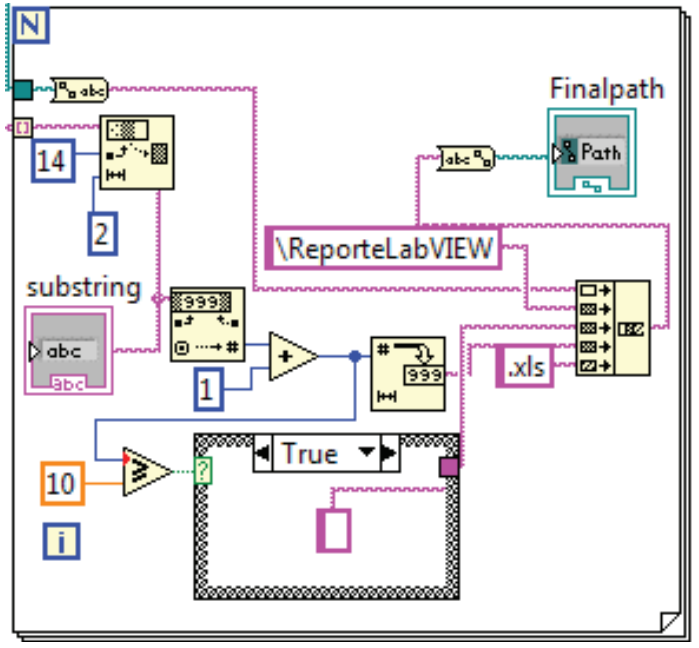

Figura 10a. Parte del código para el reporte de datos

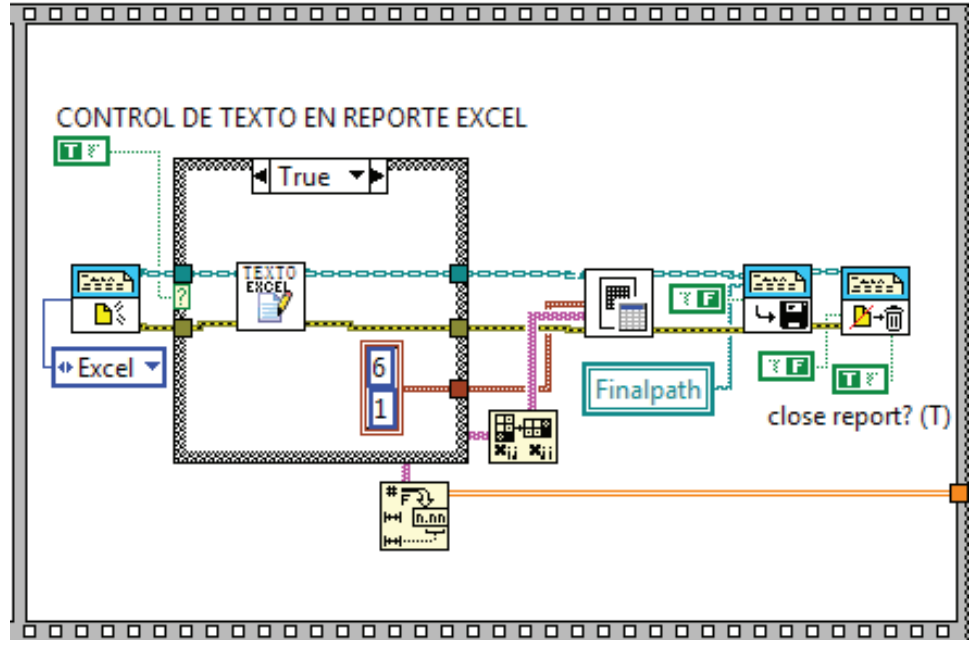

Figura 10b. Parte del código para el reporte de datos 
Tabla 2. Límites de los parámetros de la interfaz de usuario

\begin{tabular}{llll}
\hline \multicolumn{1}{c}{ Parámetro inicial } & \multicolumn{1}{c}{ Característica } & Intervalo mínimo & Intervalo máximo \\
\hline Ei $[\mathrm{V}]$ & Potencial inicial & 2 & -2 \\
Ef $[\mathrm{V}]$ & Potencial final & 2 & -2 \\
Eo $[\mathrm{V}]$ & Potencial de equilibrio & Debe estar entre Ei y Ef \\
Velocidad [V/s] & $\begin{array}{l}\text { Velocidad de barrido o } \\
\text { pendiente de la rampa }\end{array}$ & 0.001 & 2 \\
Pasos [Pasos/s] & Número de pasos por voltio & 10 & 500 \\
Tiempo de equilibrio $[\mathrm{s}]$ & $\begin{array}{l}\text { Tiempo de espera dado por el usuario } \\
\text { para que Eo se estabilice y se inicialice el } \\
\text { experimento }\end{array}$ & 0 & 300 \\
Número de ciclos & & 1 & 50 \\
\hline
\end{tabular}

En la figura 11 se observa la HMI diseñada.

Para el desarrollo de la HMI se tuvo en cuenta la Norma ISO 9241, en su parte 10, Principios de Diálogo, donde se habla del diseño ergonómico de programas para equipos con pantallas de visualización de datos; teniendo en cuenta colores, posición de los botones, posición de la pantalla, aspecto coherente que respondieran a las expectativas del usuario, indica- ciones con claridad, presentación de la información clara, etcétera, según lo plantea la norma. Se implementó una máquina de estado para detectar los valores fuera del intervalo de los parámetros de la técnica voltamétrica (figuras 12a y 12b), de tal forma que si el usuario intenta ingresar un valor fuera del intervalo establecido inmediatamente se notifica esto mediante un mensaje.

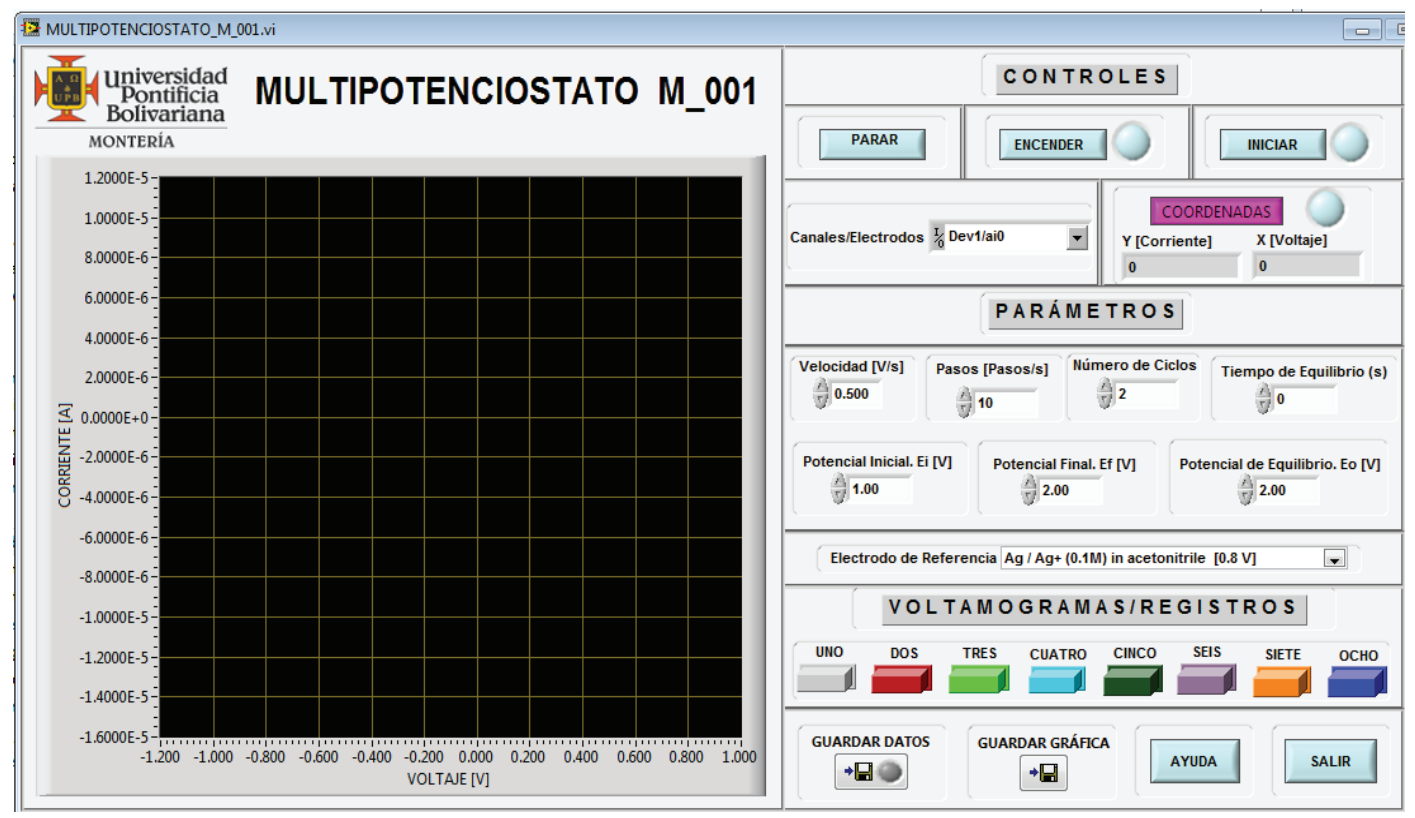

Figura 11. Interfaz de usuario del sistema multicanal 


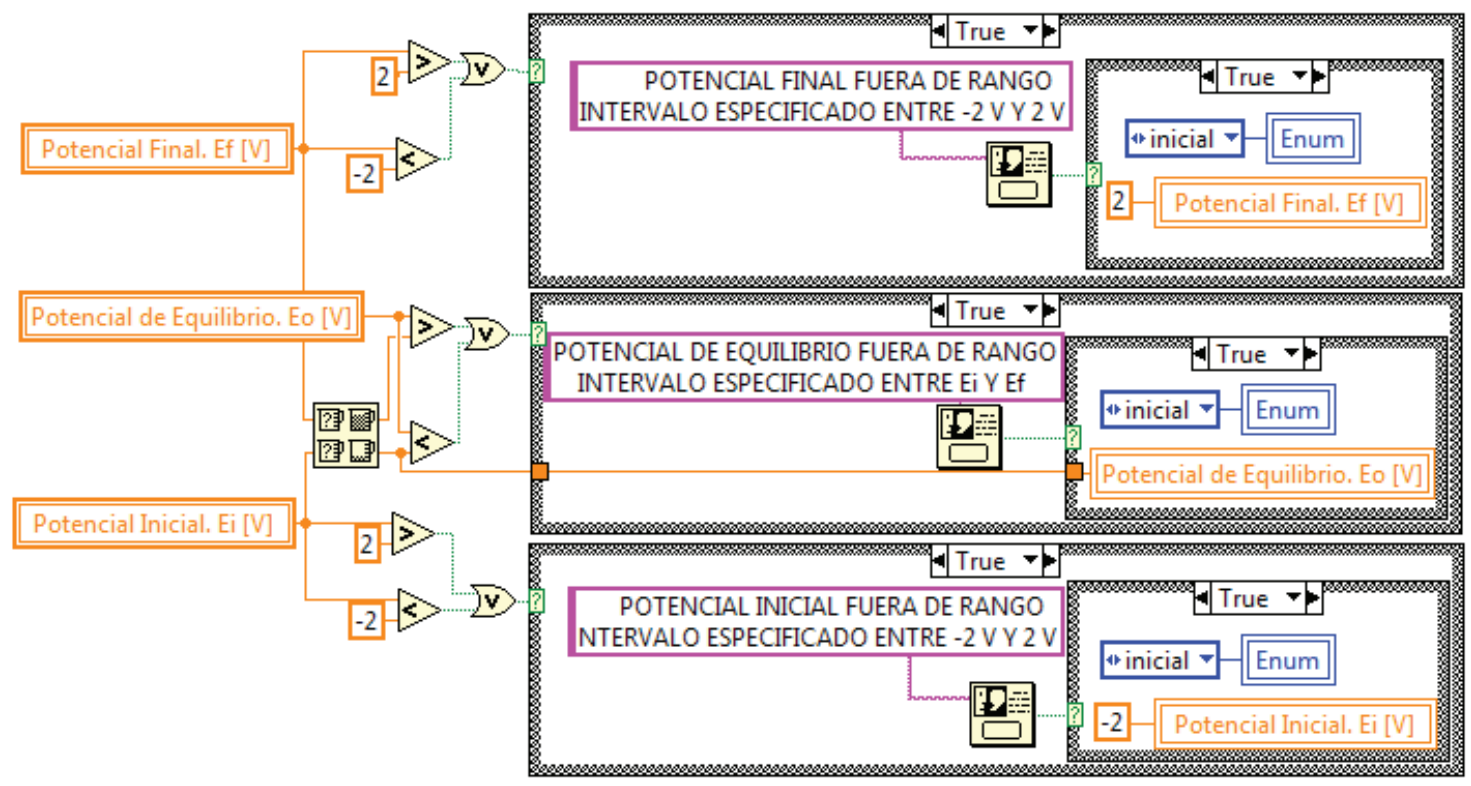

Figura. 12a. Parte de la máquina de estado

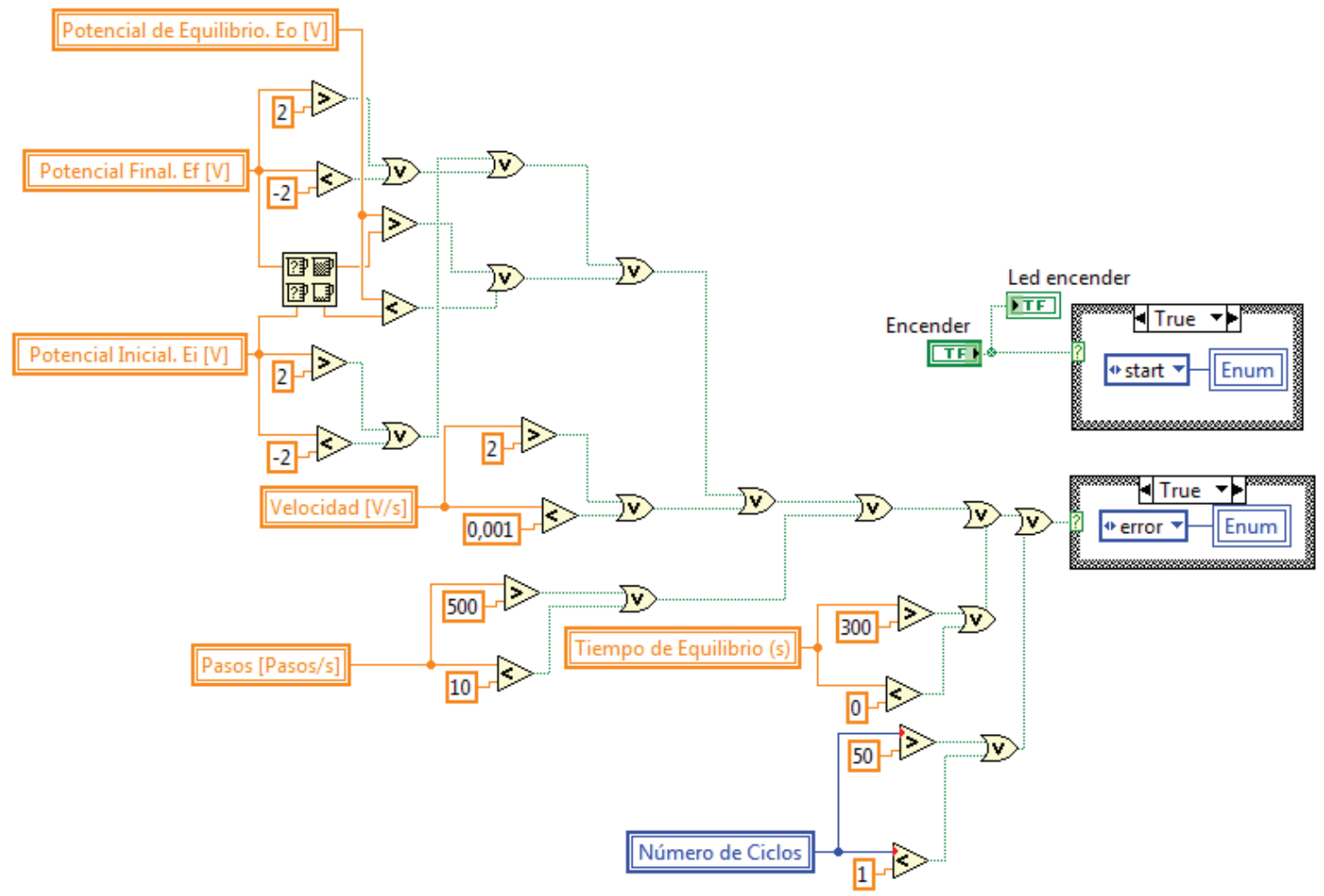

Figura. 12b. Parte de la máquina de estado 
En la HMI, los controles se refieren a los botones de encendido, paro, inicialización del experimento y la opción de escoger la cantidad de canales o electrodos de trabajo con que se quiere realizar el análisis electroquímico, el usuario puede escoger de uno a ocho canales. También, puede habilitar las coordenadas de un punto de la curva en la pantalla de la gráfica para determinar un valor específico de la curva.

Otras opciones que se presentan son los registros, donde el usuario puede guardar la gráfica obtenida, en la figura 13 se muestra parte del código que realiza esta opción.

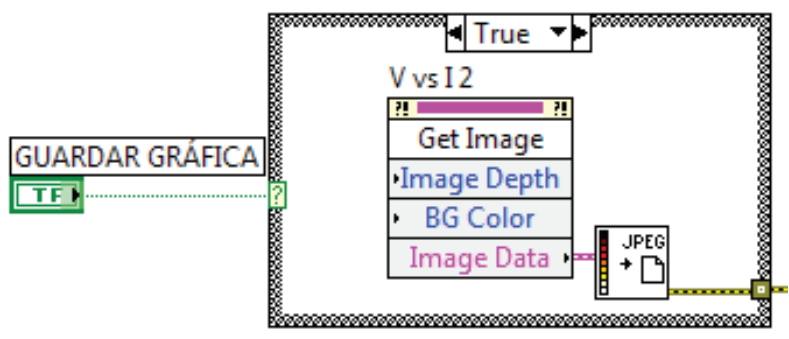

Figura 13. Parte de la programación que guardan las gráficas
El usuario también puede elegir qué voltamogramas quiere visualizar, dependiendo del número de electrodos de trabajo asignados, esto se realizó utilizando controles booleanos, uno por cada gráfica. La estructura $X Y$ Graph y sus propiedades nodales se muestran en la figura 14.

El diagrama de flujo general del programa diseñado se observa en la figura 15.

\section{Resultados y discusiones}

Al buscar las referencias bibliográficas sobre lenguas electrónicas, y específicamente los sistemas de medición, se encuentra que en todos los casos la técnica electroquímica que se utiliza se genera utilizando sistemas microcontrolados, donde obligatoriamente se tienen que utilizar dispositivos que acondicionen y acoplen las señales de voltaje generadas y de corrientes adquiridas (Alcañiz, 2011). La utilización de la instrumentación virtual y módulos de adquisición de la National Instruments permitieron un mejor acoplamiento de las señales y, por qué no decirlo, un sistema de medición más de tipo portátil.

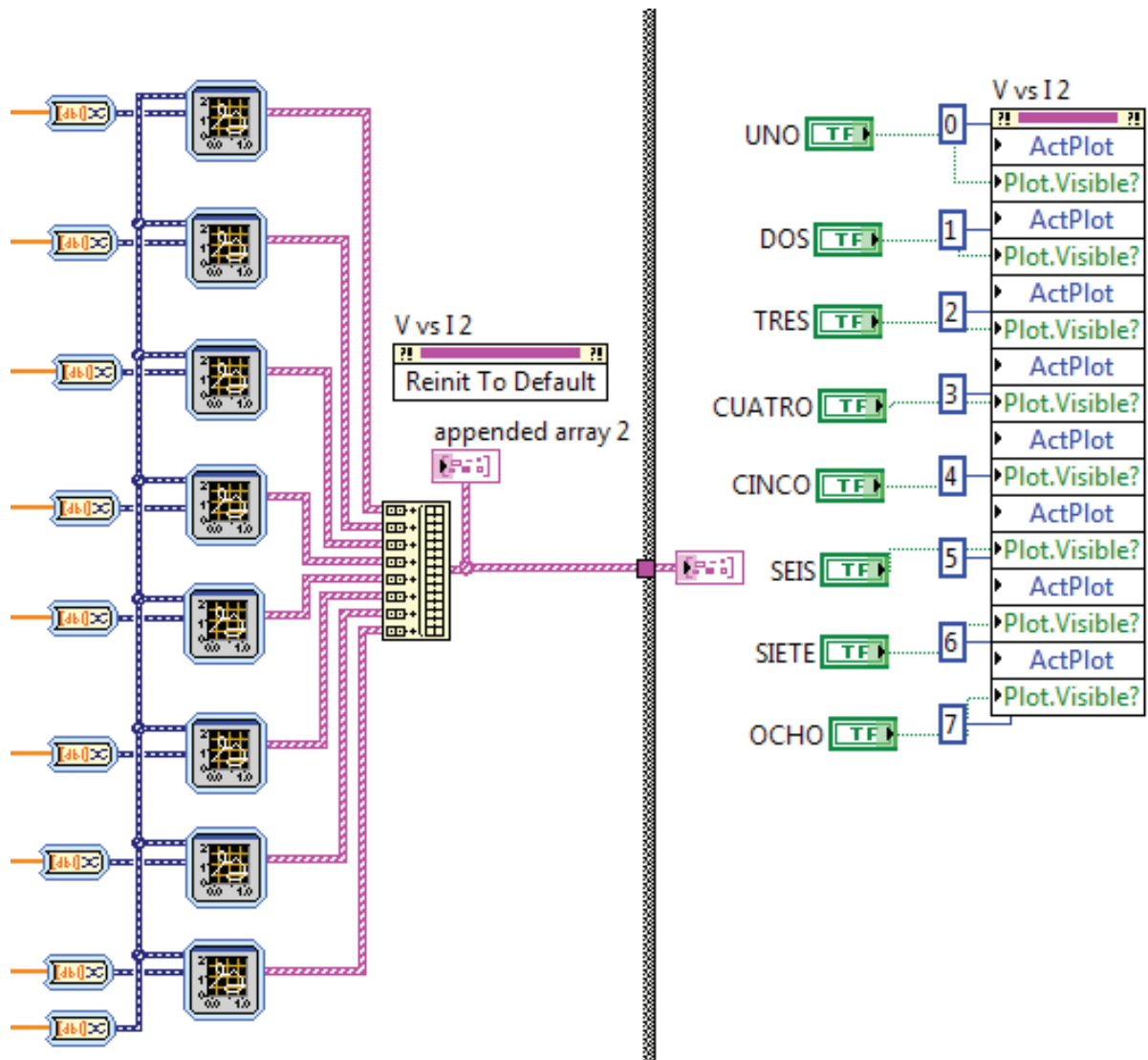

Figura 14. Estructura para los voltamogramas 


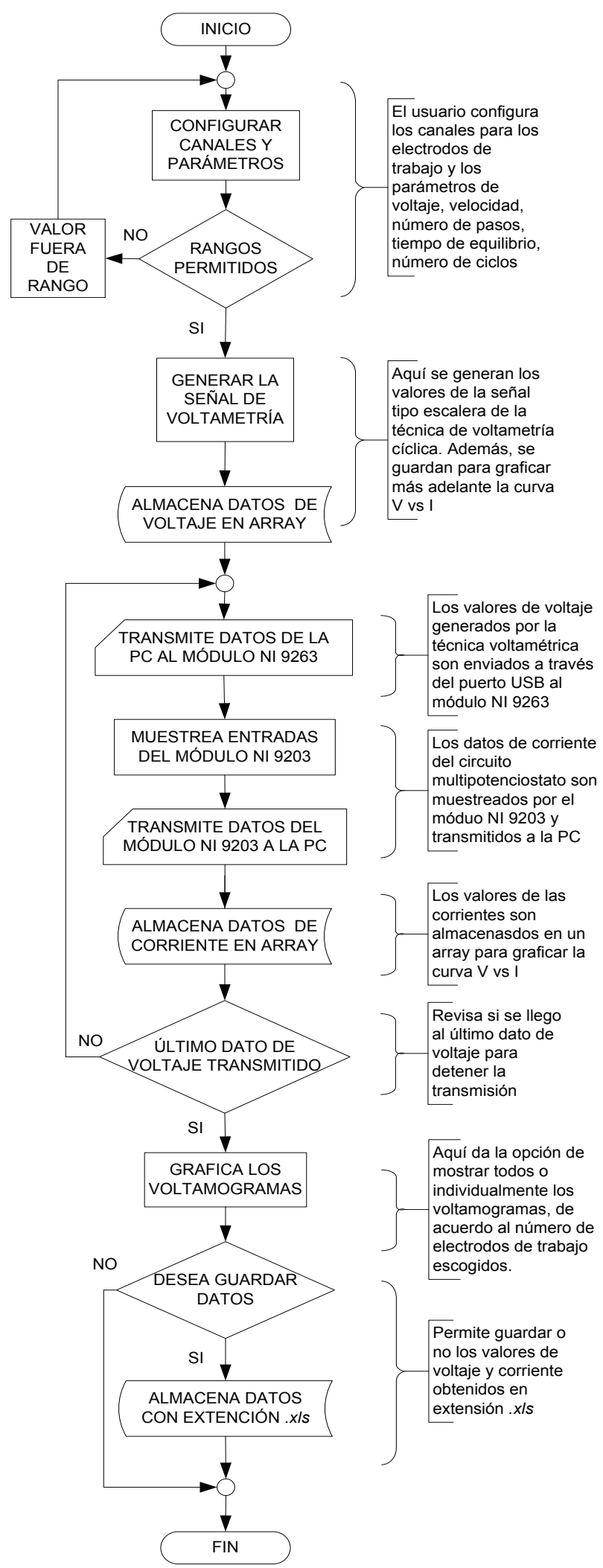

Figura 15. Diagrama de flujo del software de aplicación del sistema multicanal
El proyecto comenzó con el diseño de los VI, en lo primero que se trabajó fue en la técnica de voltametría. Una vez obtenida la señal deseada, el diseño se centró en implementar el VI para la comunicación con los módulos de adquisición de datos DAQ NI 9203 y 9263; las primeras pruebas se realizaron con un solo canal de medida para poder obtener un mejor control sobre el experimento y lograr localizar con mayor facilidad los errores que se presentaran. Se utilizaron los VI DAQ Assistant, subVI, ya prediseñados para la transmisión y recepción de los datos, y se logró realizar la comunicación con la PC y los módulos de adquisición. Fue una prueba sencilla que involucraba solo una parte del programa total, pero cuando se quiso realizar una prueba con todo el software diseñado se presentaron una serie de errores, tanto en generación de la onda de voltaje como en la forma de onda de la corriente detectada. Ambas variables fueron monitoreadas, la onda de voltaje generada con un osciloscopio y la forma de onda de corriente con ayuda de un waverform charts. En ellas se presentaban retardos de tiempo y formas de onda inconsistentes a las esperadas, de acuerdo con la sustancia que se trabajó. En las pruebas realizadas, se utilizaron electrodos de platino diseñados en los laboratorios de la Universidad Pontificia Bolivariana Seccional Montería y la sustancia ferrocianuro de potasio, con una señal voltamétrica conocida, la cual tiene unas propiedades redox bien caracterizadas en la literatura para poder comprobar el funcionamiento del dispositivo.

Al graficar el voltaje vs la corriente para poder observar si el voltamograma tenía una forma de onda típica de la técnica utilizada, no se obtuvieron los resultados que se esperaban, no generaba una forma de onda similar a la del ferrocianuro de potasio. En la figura 16 se muestra la forma de onda del voltamograma obtenida con los VI DAQ Assistant y en la figura 17 la onda típica con el tipo de técnica utilizada.

Al monitorear, analizar y simular el software se notó que la programación con los DAQ Assitant demandaba mayor capacidad de procesamiento por parte de la CPU de la PC, lo que provocaba fallas en la comunicación, especialmente en la recepción de datos. Una vez encontrado el problema se decidió trabajar el diseño de la adquisición de datos a bajo nivel, utilizando los VI DAQmx, subVI específicos para la comunicación, como el DAQmx Read, DACmx Write, DAQmx Clear Task, DAQmx start y el DAQmx Create Channel. Estos VI se configuraron tanto para la transmisión como para la recepción de datos y se simuló el programa hasta obtener los resultados satisfactorios para la comunicación. En la figura 18 se muestra el voltomograma obtenido. 


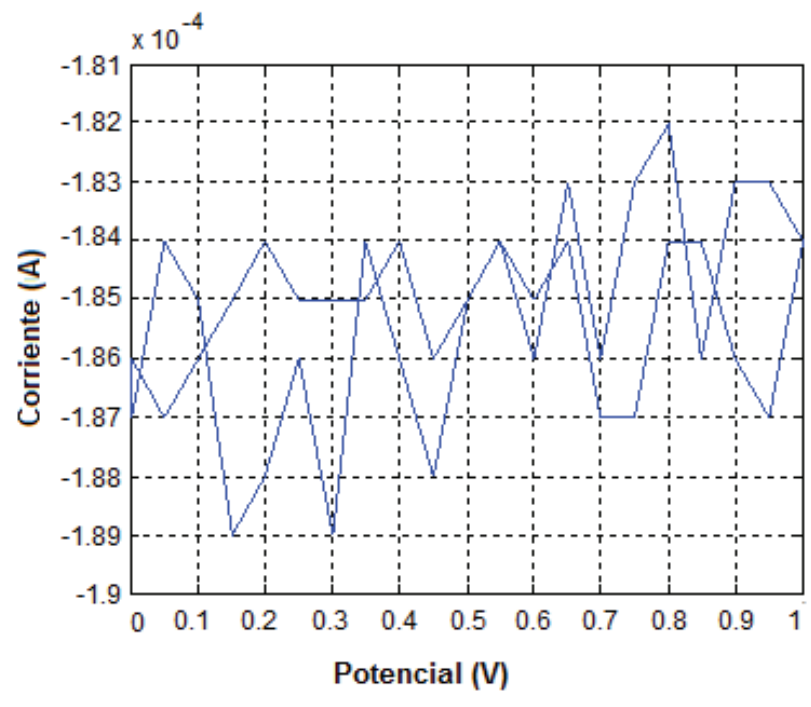

Figura 16. Voltamograma obtenidos con el VI DAQ Assitant

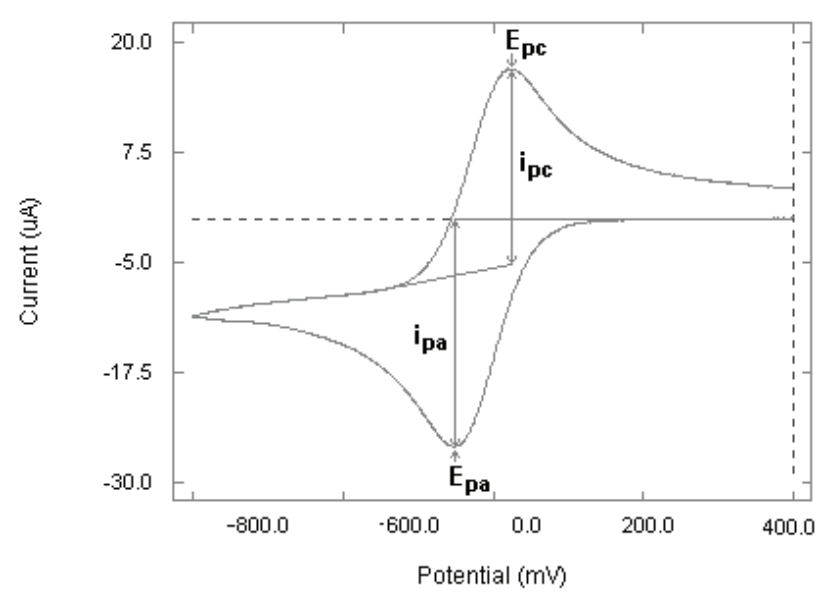

Figura 17. Gráfica de voltametría cíclica, típica del ferrocianuro de potasio (Aprende en línea, 2010)

En la figura 19 se observan los sensores poliméricos diseñados con sustrato de platino, cuya preparación se realizó en dos geometrías distintas, una cilíndrica y otra de disco, con el fin de evaluar las características electroquímicas de los sensores resultantes, su estabilidad y reproducibilidad. Los sustratos se prepararon a partir de un hilo de platino de $1 \mathrm{~mm}$ de diámetro y $7 \mathrm{~mm}$ de longitud, soldado a un alambre de cobre de $12 \mathrm{~cm}$ de longitud.

Los resultados de la figura 18, se compararon utilizando la misma sustancia, con los datos arrojados por

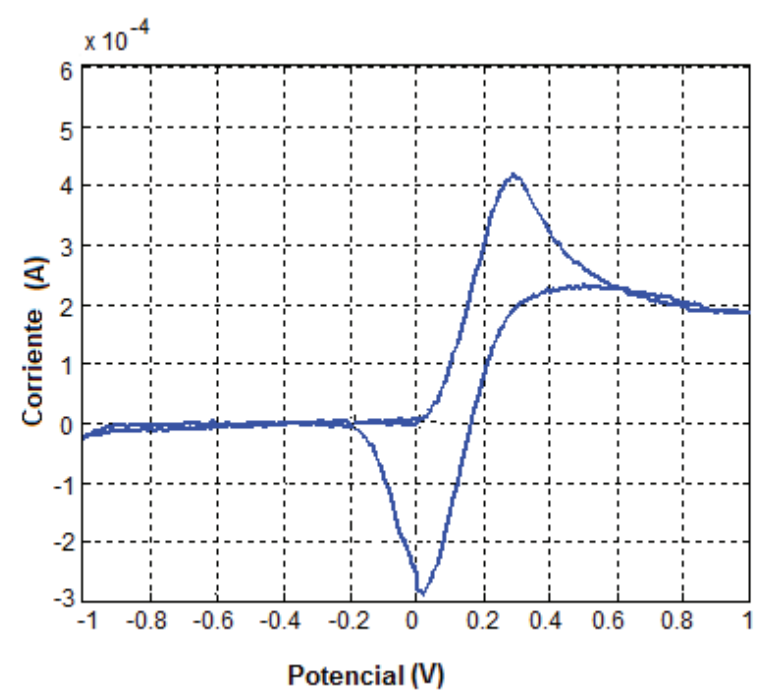

Figura 18. Voltamograma utilizando ferrocianuro de potasio

el potenciostato comercial PARSTAT 2263, estandarizado en el mercado mundial y que cumple con todas las especificaciones de este tipo de equipos. La figura 20 muestra la forma de onda utilizando el PARSTAT 2263. Al comparar las figuras 17, 18 y 20 se observa similitud en las señales, lo que indicaba el correcto funcionamiento del multipotenciostato diseñado, al comprobar la posición de los picos de oxidación y reducción de la solución en determinado potencial dado.

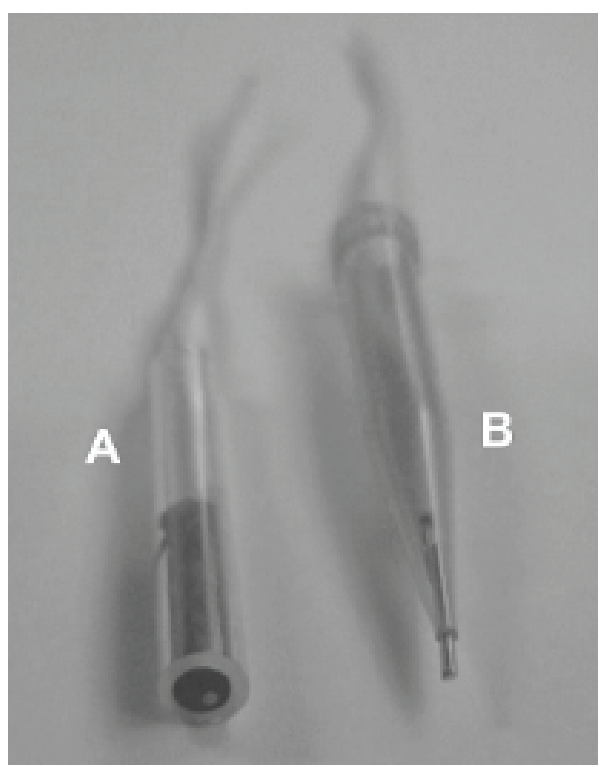

Figura 19. Sustratos de platino empleados en la elaboración de sensores poliméricos: A. De disco; B. Cilíndrico 


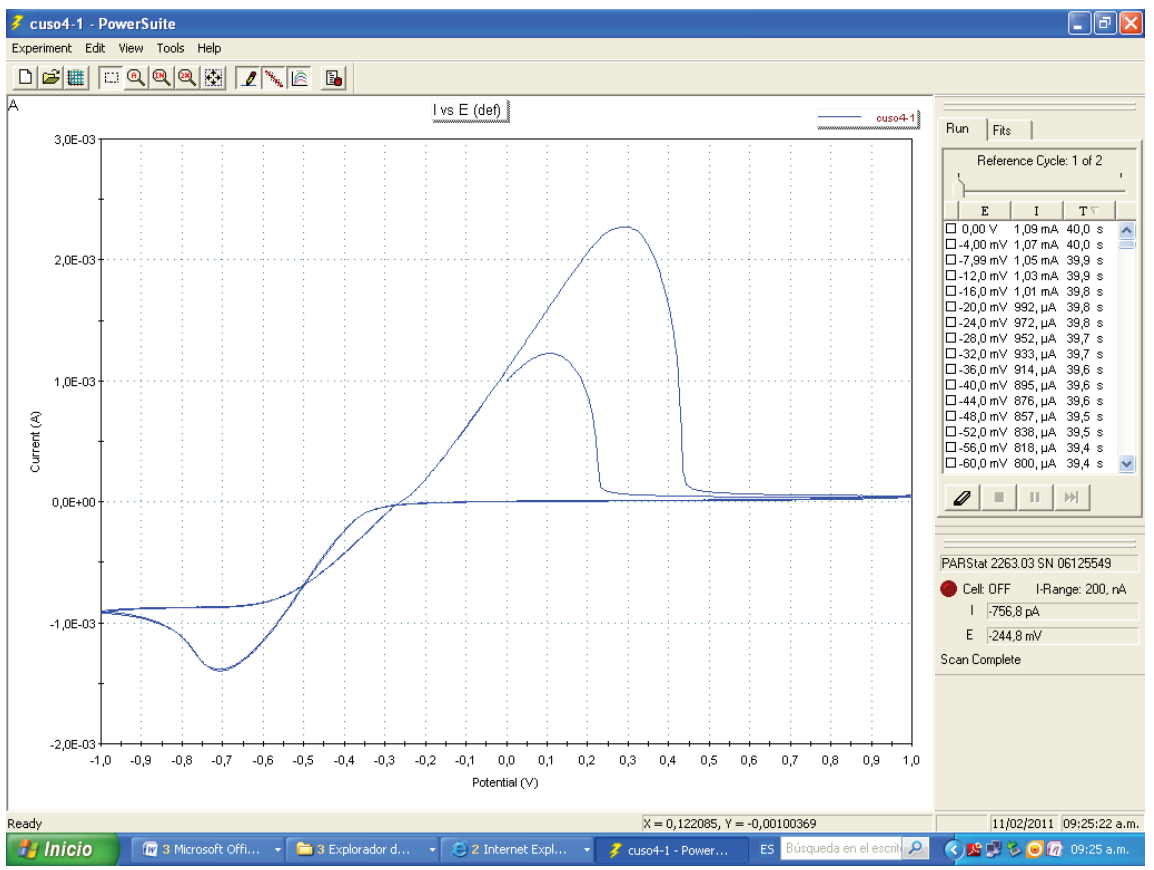

Figura 20. Voltamograma utilizando el PARSTAT 2263
Al analizar los datos adquiridos se observa que para cumplir la ley de Ohm, cuando se suministra una señal de voltaje negativa se obtiene una señal de corriente negativa, asimismo al entregar una señal positiva de voltaje se obtiene una señal positiva de corriente, lo cual no se cumple en la primera prueba cuando se utilizó el VI DAQ Assistant (figura 16). Además, en la medición de procesos que involucran reacciones electroquímicas de óxido-reducción, la impedancia interna de la celda varía con respecto al voltaje aplicado, luego la impedancia deja de ser un parámetro constante y pasa a ser un parámetro variable. Así se logran ver variaciones no lineales de la corriente con respecto al voltaje como se muestran en la figura 17 y 18 . En la figura 21 se muestran las gráficas de voltamogramas cuando se utilizan los ocho canales del NI9203.

Con este dispositivo no solo se pueden realizar medidas simultáneas con la red de sensores, también es posible observar que el dispositivo de medidas multicanal diseñado cumple con los objetivos planteados inicialmente.

Adicionalmente, el sistema multicanal se utilizó para realizar un análisis de algunas muestras comerciales de café (la Bastilla, Córdoba, Almendra Tropical, Sello Rojo y Universal) preparadas según la norma NTC 3566 (preparación de muestras para uso en análisis sensorial); se pesó y preparó una muestra de $7.0 \mathrm{~g}$ de café en $100 \mathrm{~mL}$ de agua, la cual fue calentada hasta el punto de ebullición. Después, se vertió en un beaker dejando decantar la infusión durante 5 min. Luego se eli- minaron los residuos de la superficie de la bebida y se la dejó enfriar hasta alcanzar la temperatura ambiente.

Cada sensor mostró una respuesta electroquímica particular hacia cada una de las muestras de café. Los datos obtenidos con las señales registradas con la red de sensores se utilizaron para realizar la clasificación de las muestras de acuerdo con su naturaleza química.

La red de sensores fue elaborada en los laboratorios de la UPB a partir de electropolimerización de pirrol (PPy) con diferentes agentes dopantes. Las muestras de café se midieron de forma aleatoria con el sistema mul-

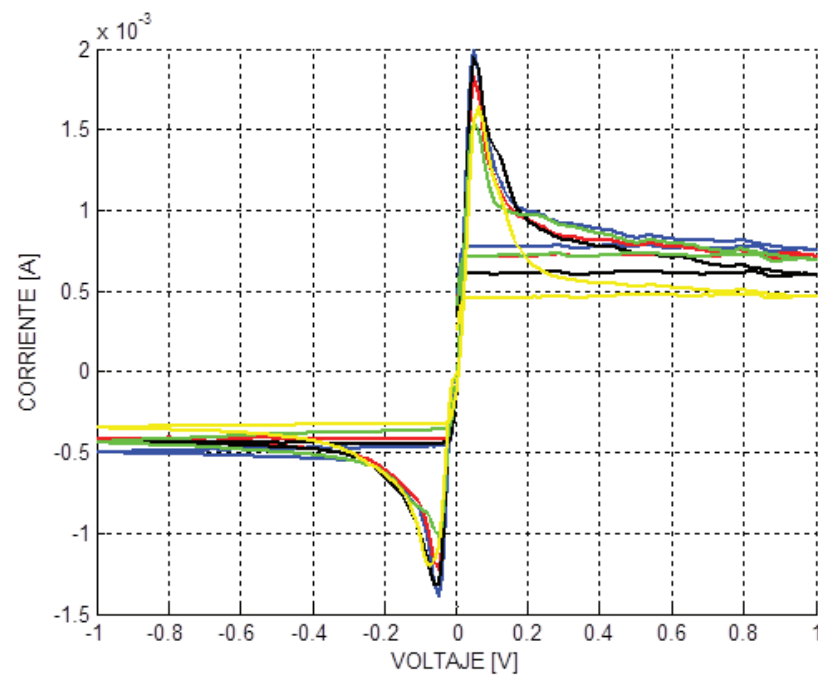

Figura 21. Voltamograma utilizando varios canales activos 
(a)

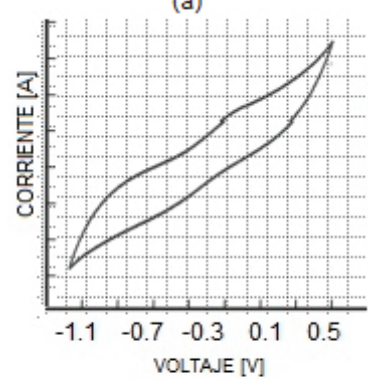

(d)

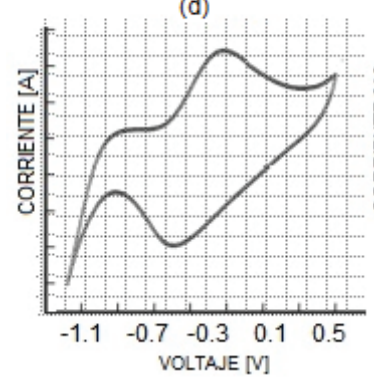

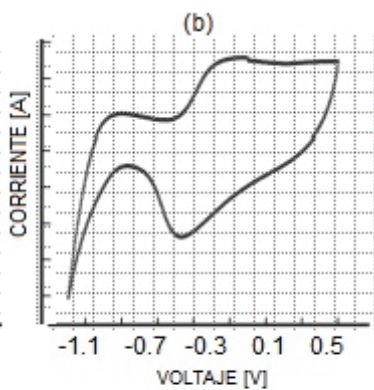

(e)

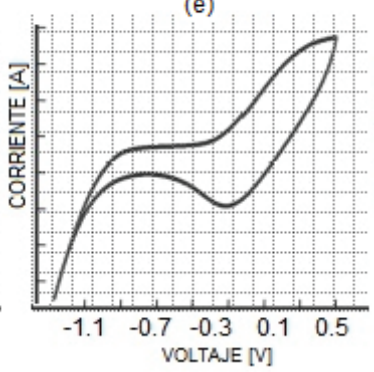

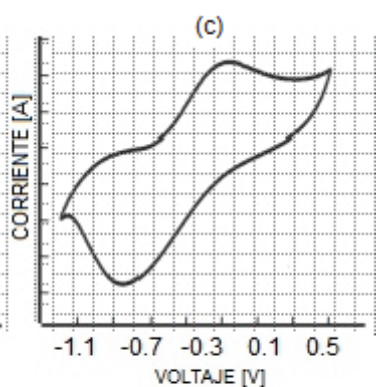

(f)

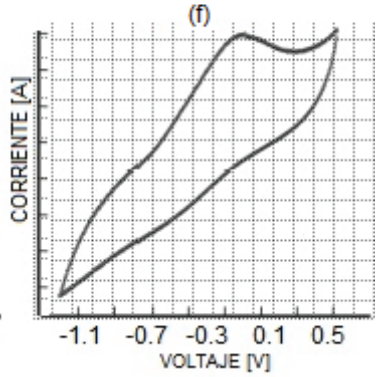

Figura 22. Voltamograma registrado con el sistema multicanal diseñado a diferentes muestras de café Córdoba: a) PPy/AQDS, b) PPy/DBS, c) PPy/FCN, d) PPy/HCF, e) PPy/PWA y f)PPy/PTS ticanal. Una vez preparadas dichas muestras se procedió a realizar el experimento en un intervalo de potencial de $-1.0 \mathrm{~V}$ a $0.5 \mathrm{~V}$ con una velocidad de barrido de $100 \mathrm{mV} / \mathrm{s}$. Para cada medida fue necesario realizar inicialmente 10 voltamperogramas para estabilizar las señales. A cada muestra se le realizaron ocho medidas para probar la repetitividad del método.

El análisis estadístico de los datos se realizó con la técnica multivariante PCA (Principal Component Analysis), que permite visualizar matrices de datos con muchas variables de entradas y reducirla a 2 o 3 variables, las cuales contienen la máxima varianza o información.

Cabe destacar que con la red de sensores diseñada fue posible conseguir señales voltamétricas con picos bien definidos, a diferencia de la que se obtuvo con el uso de los sensores voltamétricos metálicos tradicionales utilizados por otros autores (Tian et al., 2007; Söderström et al., 2003).

En la figura 22 se muestra la respuesta de los sensores frente a cada muestra de café. La variedad de respuesta de los sensores evidencia la alta selectividad cruzada de la red. Además, se demuestra el buen funcionamiento del sistema multicanal diseñado. De esta manera, los datos suministrados por las señales electroquímicas de la red de sensores pueden ser utilizados para diferenciar y clasificar las muestras de café sin necesidad de analizar sus componentes particulares.

El sistema trabaja con diferentes cantidades de sensores que van desde uno (1) hasta un máximo de ocho (8). Esta cantidad de canales es suficiente para que el dispositivo registre señales típicas de una lengua elec- trónica, en la actualidad cuando se trabaja con señales voltamétricas, por lo general se utilizan 6 sensores.

\section{Conclusiones}

El sistema multicanal diseñado es un dispositivo que permite realizar análisis de reacciones electroquímicas mediante la técnica de voltametría cíclica. Fue posible establecer parámetros puntuales de potenciales aplicados a las sustancias utilizadas, lo que permitió tener más control sobre las prestaciones del dispositivo y tener un mejor acoplamiento con la red de sensores y el software de control y tratamiento de datos. Por esto, el dispositivo multicanal diseñado puede llegar a tener más presencia en los diferentes procesos industriales, debido al desarrollo científico y tecnológico que estos sistemas de medida están alcanzando.

En el sistema multicanal diseñado se pueden definir los intervalos de suministro de potenciales a la celda electrolítica (Eo, Ei y Ef), la velocidad y número de pasos aplicados dependiendo de la aplicación para el estudio de experimentos específicos. Esto influye directamente en el análisis del experimento, porque las corrientes obtenidas para cada intervalo dado varían, por lo que se generan datos diferentes en cada análisis.

Es importante resaltar que para el sistema multicanal desarrollado, al utilizar los módulos NI 9203, NI 9263 y el CompactDAQ USB 9174 de la National Instruments, el acoplamiento entre el software de aplicación y el circuito multipotenciostato básico se hizo de manera más fácil y no fue necesario utilizar diferentes circuitos integrados 
como microprocesador o microcontroladores, conversores análogo digital y digital análogo, ya que vienen integrados o embebidos en las mismas tarjetas de adquisición. Con base en lo expuesto anteriormente se logró así un sistema más liviano y pequeño que los encontrados en el comercio y, a la vez, con especificaciones similares gracias a sus características eléctricas.

También cabe resaltar que la utilización del software Labview facilita y permite una mejor programación de la HMI, llevando a cabo la tarea solicitada, en este caso la interfaz de la lengua electrónica, de una forma más concreta y versátil. De la misma manera, permitió la comunicación con los módulos. Otra ventaja de utilizar el LabVIEW y los módulos de adquisición de National Instruments es que permitió generar la señal de voltaje necesaria para la aplicación de la técnica utilizada sin necesidad de generar este voltaje por medio de un dispositivo externo.

Los sistemas de lenguas electrónicas, con ayuda de la instrumentación virtual, tienen un futuro prometedor, gracias a las novedosas técnicas de medición que han tenido y pueden llegar a tener, pues las investigaciones en este campo presentan notables resultados y más aún con la incursión de nuevos materiales en la fabricación de sensores, nueva programación y nueva instrumentación para la adquisición y tratamiento de datos. Los grupos de investigación de nuevos materiales DANM (desarrollo y aplicación de nuevos materiales) y de electrónica GIE (grupo de investigación en electrónica) de la Universidad Pontificia Bolivariana seccional Montería, seguirán trabajando en esta línea para lograr un sistemas aún más portátil, claro está con las características y rendimiento de los sistemas comerciales, pero aún más específico para determinadas tareas.

\section{Agradecimientos}

Los autores agradecen el soporte financiero brindado por el Departamento Administrativo de Ciencia, Tecnología e Innovación Colciencias (Colombia).

\section{Referencias}

Alcañiz M. Diseño de un sistema de lengua electrónica basado en técnicas electroquímicas voltamétricas y su aplicación en el ámbito agroalimentario, Universidad Politécnica de Valencia, Departamento de Ingeniería Electrónica, 2011, pp. 93-100. Tomado de Aprende en línea [en línea] [fecha de consulta: agosto de 2010]. Disponible en: http://aprendeenlinea.udea.edu. $\mathrm{co} / \mathrm{lms} / \mathrm{moodle} / \mathrm{mod} / \mathrm{resource} / \mathrm{view} \cdot \mathrm{php}$ ?id=54362.

Analog Devices. Next Generation OP07 Ultralow Offset Voltage Operational Amplifier, OP77 [en línea] [fecha de consulta: 20 de abril de 2010]. Disponible en: http://www.datasheetcata$\log . \mathrm{com} /$.

Arrieta A., Osorio. K. XXIX Congreso latinoamericano de química - XVI Congreso colombiano de química, en: VI Congreso colombiano de cromatografía, 29, 2010, Colombia. Sensores electroquímicos de polipirrol como unidades sensibles de una lengua electrónica para el análisis de café.

Arrieta A., Tarazona. R. Study of Kinetic Formation and the Electrochemical Behavior of Polypyrrole Films. Journal of the Chilean Chemical Society, volumen 54, julio de 2008: 1568-1573.

Calobozo J. Realización de un potenciostato, ingeniería técnica industrial, especialidad en técnica industrial, Universitat Rovira i Virgili, Departamento de Ingeniería Electrónica y Automática, noviembre 2004, pp. 11-16.

Gardner J., Shin H., Hines E., Dow C. An Electronic Nose System for Monitoring the Quality of Potable Water. Sensors and Actuators B. Chemical, volumen 69, octubre de 2000: 336-341.

Ivarsson P., Kikkawa Y., Winquist F., Krantz-Rülcker Ch., Höjer N., Hayashi K., Toko K., Lundströma I. Comparison of a Voltammetric Electronic Tongue and a Lipid Membrane Taste Sensor. Analytica Chimica Acta, volumen 449, julio de 2001: 59-68.

Jiménez C., Muñoz F., Bratov A. y Domínguez. C. I encuentro internacional ciencias sensoriales y de la percepción 2002, Bellaterra, Barcelona. Lenguas electrónicas: sensores químicos aplicados a la medida del sabor y al control de calidad de los alimentos, Instituto de Microelectrónica de Barcelona (IMBCNM), 2002.

Kounave S. Voltammetric Techniques, en: Frank Settle, ed. Handbook of Instrumental Techniques for Analytical Chemistry, United States of America, Prentice Hall, 1997, capítulo 37, pp. 709-725.

Lajara J., Pelegrí J. LabVIEW entorno gráfico de programación, libro Labview, Barcelona, España, Marcombo, 2007.

Lvova L., Shin S., Legin A., Vlasov Yu., Soo J., Sig G., Nam.H. AllSolid-State Electronic Tongue and its Application for Beverage Analysis. Analytica Chimica Acta, volumen 468, julio de 2001: 303-314.

Natale C., Macagnano A., Martinelli E., Paolesse R., Proietti E., D'Amico A. The Evaluation of Quality of Post-Harvest Oranges and Apples by Means of an Electronic Nose. Sensors and Actuators B. Chemical, volumen 78, agosto de 2001: 26-31.

National Instruments Datasheet. User Guide and Specifications NI cDAQ-9178/9174, National Instruments Corp., octubre, 2009a.

National Instruments Datasheet. Operating Instructions and Specifications NI 9263, National Instruments Corp., febrero, 2009b.

National Instruments Datasheet. Operating Instructions And Specifications NI 9203, National Instruments Corp., febrero, 2008.

Parra V., Arrieta Á., Fernández-Escudero J., García H., Apetrei C., Rodríguez M., De Saja J. E-Tongue Based on a Hybrid Array of Voltammetric Sensors Based on Phthalocyanines, Perylene Derivatives and Conducting Polymers: Discrimination Capa- 
bility Towards Red Wines Elaborated with Different Varieties of Grapes. Sensors and Actuators B: Chemical, volumen 115, octubre de 2005: 54-61.

Parra V., Arrieta Á., Fernández-Escudero J., Rodríguez-Méndez M., De Saja. J. Electronic Tongue Based on Chemically Modified Electrodes and Voltammetry for the Detection of Adulterations in Wines. Sensors and Actuators B: Chemical, volumen 118, mayo de 2006a: 448-453.

Parra V., Arrieta Á., Fernández-Escudero J., Íñiguez M., De Saja J., Rodríguez-Méndez M. Monitoring of the Ageing of Red Wines in Oak Barrels by Means of an Hybrid Electronic Tongue. Analytica Chimica Acta, volumen 563, octubre de 2006b: 229-237.

Persaud K., Dodd G. Analysis of Discrimination Mechanisms in the Mammalian Olfactory System Using a Model Nose Nature, volumen 229, septiembre de 1982: 352-355.

Pividori M. Nuevos genosensores amperométricos, diseño y construcción, (tesis de doctorado), Universidad Autónoma de Barcelona, 2002 [en línea][fecha de consulta: 20 de abril de 2010]. Disponible en: http://ddd.uab.cat/pub/tesis/2002/tdx-1122104173753/msp1de4.pdf

Rudnitskaya A., Polshin E., Kirsanov D., Lammertyn J. Instrumental Measurement of Beer Taste Attributes Using an Electronic Tongue. Analytica Chimica Acta, volumen 646, mayo de 2009: 111-118.

Söderström C., Borén H., Winquist F., Krantz-Rülcker C. Use of an Electronic Tongue to Analyze Mold Growth in Liquid Media. International Journal of Food Microbiology, volumen 83, junio de 2003: 253-261.

Tian Shi-Yi, Deng Shao-Ping, Chen Zhong-Xiu. Multifrequency Large Amplitude Pulse Voltammetry: a Novel Electrochemical Method for Electronic Tongue. Sensors and Actuators B, volumen 123, mayo de 2007: 1049-1056.
Toko K. Electronic Sensing of Tastes. Electroanalysis, volumen 10, diciembre de 1998: 657-669.

Verrelli G., Francioso L., Paolesse R., Siciliano P., Di Natale C., D'Amico A., Logrieco A. Development of Silicon-Based Potentiometric Sensors: Towards a Miniaturized Electronic Tongue. Sensors and Actuators B: Chemical, volumen 123, abril de 2007: 191-197.

Vlasov Y., Legin A., Rudnitskaya A. Electronic Tongues and Their Analytical Application. Analytical and Bioanalytical Chemistry, volumen 373, abril de 2001: 136-146.

Vlasov Y., Legin A. Non-Selective Chemical Sensors in Analytical Chemistry: from "Electronic Nose" to "Electronic Tongue. Fresenius' Journal of Analytical Chemistry, volumen 361, febrero de 1998: 255-260.

Winquist F., Lundstr"om I., Wide P. The Combination of an Electronic Tongue and an Electronic Nose. Sensors and Actuators B: Chemical, volumen 58, septiembre de 1999: 512-517.

Winquist F., Wide P., Lundströma I. An Electronic Tongue Based on Voltammetry. Analytica Chimica Acta, volumen 357, diciembre de 1997: 21-31.

\section{Este artículo se cita:}

\section{Citación estilo Chicago}

Arrieta-Almario, Álvaro Angel, Rosa Liliana Tarazona-Cáceres. Sistema multipotenciostato basado en instrumentación virtual. Ingeniería Investigación y Tecnología, XV, 03 (2014): 321-337.

\section{Citación estilo ISO 690}

Arrieta-Almario A.A, Tarazona-Cáceres R.L. Sistema multipotenciostato basado en instrumentación virtual. Ingeniería Investigación y Tecnología, volumen XV (número 3), julio-septiembre 2014: 321-337.

\section{Semblanzas de los autores}

Álvaro Angel Arrieta-Almario. Licenciado en química y biología por la Universidad de Córdoba/Montería. Phd. en la Universidad de Valladolid, estudios en química avanzada, diseño de una red de sensores electroquímicos inespecíficos basados en polímeros conductores para el análisis sensorial de vinos (sensores de lengua electrónica) (enero de 2005). Postdoctorado Universidad de Valladolid (junio de 2008). Docente interno en la Universidad Pontificia Bolivariana Seccional Montería. Es coordinador del grupo de investigaciones de nuevos materiales DANM/ desarrollo y aplicación de nuevos materiales de la Universidad Pontificia Bolivariana Seccional Montería. Coordinador de Investigaciones CIDI. Consejero Nacional de Ciencia Tecnología e Innovación en la convocatoria 530 de Colciencias.

Rosa Liliana Tarazona-Cáceres. Ingeniera electrónica de la Universidad Pontificia Bolivariana Seccional Bucaramanga (marzo de 2000). Especialista en automática por la Universidad Pontificia Bolivariana Medellín (junio de 2009). Candidata a magister de la Universidad Pontificia Bolivariana Medellín. Docente interna de la Facultad de Ingeniería Electrónica de la Universidad Pontificia Bolivariana Seccional Montería. Integrante del grupo de investigaciones de nuevos materiales DANM de UPB Montería, coordinadora del grupo de investigación en ingeniería electrónica de UPB Montería GIE y coordinadora del semillero de automatización y control SINAC de la Facultad de Ingeniería Electrónica UPB Montería. 